\title{
Foreword by the Editor-in-Chief
}

\author{
Chi-Wang Shu ${ }^{1}$
}

Published online: 7 March 2019

(c) Shanghai University 2019

This is the first issue of the new, English only version of Communications on Applied Mathematics and Computation (CAMC). CAMC has a long history of publishing papers in applied and computational mathematics. It was founded in 1987 by the late Professor Benyu Guo, a prominent numerical analyst working on spectral methods and high-order finite difference methods at Shanghai University and Shanghai Normal University. In the transition to the English only version, a new editorial board has been formed, expanding in its international representation as well as coverage in more areas within applied and computational mathematics. The cooperation with Springer also ensures professional manuscript handling and distribution services.

Communications on Applied Mathematics and Computation publishes high-quality research papers and review papers in applied analysis, mathematical modeling, numerical analysis, and scientific computing. Papers involving two or more areas in this list are particularly welcome, which will hopefully distinguish this journal from others. However, high-quality papers in one of these areas are also welcome.

This first issue of the new, English only version of CAMC is a focused issue dedicated to the memory of its founding Editor-in-Chief, Professor Ben-yu Guo. We expect this focused issue to contain three parts, with papers covering a wide spectrum of applied and computational mathematics by the authors from many countries including China, Italy, Singapore, Spain, Switzerland, and United States. This focused issue serves as a good sample of the type of papers we are envisioning in the new version of CAMC.

On behalf of the editorial board, I would like to encourage potential authors to submit papers to CAMC. We will do our best to ensure timely and high-quality reviews for all the received manuscripts. We look forward to a good future of CAMC.

Chi-Wang Shu

shu@dam.brown.edu

1 Division of Applied Mathematics, Brown University, Providence, RI 02912, USA 